\title{
How Connected Autonomous Vehicles Would Affect Our World? - A Literature Review on the Impacts of CAV on Road Capacity, Environment and Public Attitude
}

\author{
Shuya Zong1, a \\ ${ }^{1}$ Department of Civil and Environment Engineering, Hong Kong Polytechnic University
}

\begin{abstract}
With the rapid development of technology, connected autonomous vehicle (CAV) is getting close to the reality. The application of CAV causes changes to road capacity, gas emission, public attitude and other realms. Lots of efforts have been spent in quantifying the potential changes and this paper is an attempt to review the relevant researches. There will be three sections, presenting review of the impacts on road capacity, environment and public attitude respectively. There is a large amount of papers making models to predict future road capacity with various penetration rate of CAV and they obtain quite different interesting results. To predict the future condition more properly, more stochastic models should be proposed. In terms of influence on environment, it may be hard to conclude whether CAV will exacerbate or relieve global warming by looking at current researches. It would be valuable to conduct a quantitative analysis on this issue. For the public attitude, this paper mainly focus on whether people are willing to use CAV and future efforts that may help with the promotion of CAV.
\end{abstract}

\section{Introduction}

Connected and automated vehicle (CAV) has aroused interest in the academic field as well as in automobile companies. It is predicted that the amount of connected cars sold will become more than 152 million in the world by 2020 [1]. $20-40 \%$ of vehicles are expected to be automated by 2030 and the full penetration could be realistic in few decades [2]. CAV is featured by its easier access to timely information, strong data processing ability and precision control. Due to these features of $\mathrm{CAV}$, the reaction time and the distance between vehicles can be reduced significantly. As a result, the application of CAV increases driving safety, comfort and potential revolution in transportation framework. Understanding the impacts of CAV will be beneficial to future transportation control, policy making and urban design. Considerable research efforts have been devoted to the impact of CAV on road capacity, traffic jam, environment and public attitude. By using different analytical tools and data collection approaches, they provide interesting anticipation of the future of CAV application. This paper will attempt to review the relevant research to capture the potential influence of CAV on the road capacity, environment and public attitude. This paper will also propose the future research potential by looking at the limitations of current researches.

There will be three sections in this paper. The first chapter discusses the influence of road throughput with different penetration rate of CAV. The second chapter then explore how environment will be affected by CAV

\footnotetext{
${ }^{\mathrm{a}}$ Corresponding author: sue.zong@ connect.polyu.hk
}

and the third chapter asks if the public will accept CAV with positive responses. Gaps and limitations of current work will also be discussed in each chapter, providing ideas for future researches.

\section{Impacts on road capacity}

Emerging CAV technologies have tremendous potential to improve traffic efficiency, safe and stability. To further apply the technologies in traffic control and planning, it is critical to evaluate how the road capacity would be improved with increasing penetration rate of CAV [3]. The research methods are either setting theoretical framework of road capacity (e.g. [4], [5]) or making simulation of CAV penetrated flow (e.g. [6], [7]).

A theoretical formulation for physical lane capacity of a single-lane road considering AV (automated vehicle) penetration rate, vehicle spacing and platoon size is derived by Chen et al in [4]. It was then found that a strict segregation of AV and RV (RV: regular vehicle) would result in lower capacity while mixed-use would realize higher capacities. The results can be illustrated in the figure 1 below. The formulation is extended further to multi-lane highway and it was found that AV should be used in most efficient lanes to maximum the road capacity. Chen et al developed a mixed routing equilibrium model to describe the flow of $\mathrm{AV}$ and $\mathrm{CV}$ with the presence of AV zones in [4]. They showed that an optimal deployment of AV zone should be applied to maximize the positive effect of $\mathrm{AV}$ and $\mathrm{CV}$ on road 
capacity. Based on [4], Ghiasi in [8] proposed an analytical framework with a Markov chain representation of spatial distribution of heterogeneous headway on the highway. This framework provides a useful tool to examine the capacity change under different autonomous vehicle penetration.

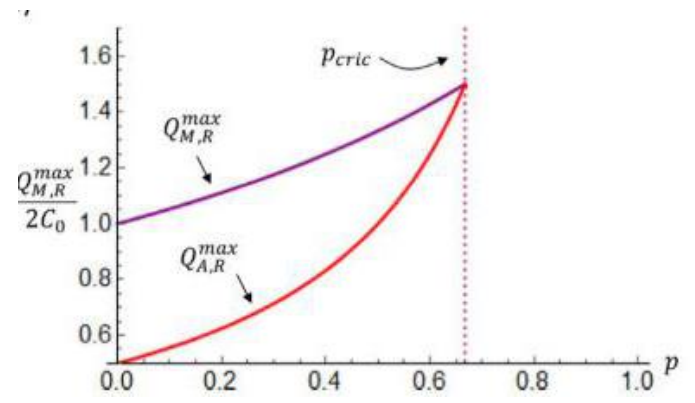

Figure 1. Mixed-use of AV and RV realizes higher capacity. $Q_{A, R}^{\max }$ :maximum capacity under the policy where AV and RV are separated. $Q_{M, R}^{\max }$ :maximum capacity under mixed use. P: AV penetration rate[4]

Apart from theoretical research, there are papers modelling the traffic flow after penetration of CAV(e.g. [9], [10], [11]). The change in road capacity depends highly on the proportion of autonomous cars: Levin and Boyles in [9] found that the proportion of AV should not exceed $75 \%$ to avoid the negative effects of inefficiency caused by mixed traffic based on a shared intersection model. Talebpour and Mahmassani in [10] analysed the effect of autonomous vehicles and connected vehicles individually and suggested that the throughput will increase if the penetration rate is medium. Moreover, they come up with a model describing the combined effects of those two types of cars. The results is explicitly illustrated in the figure 2 below.

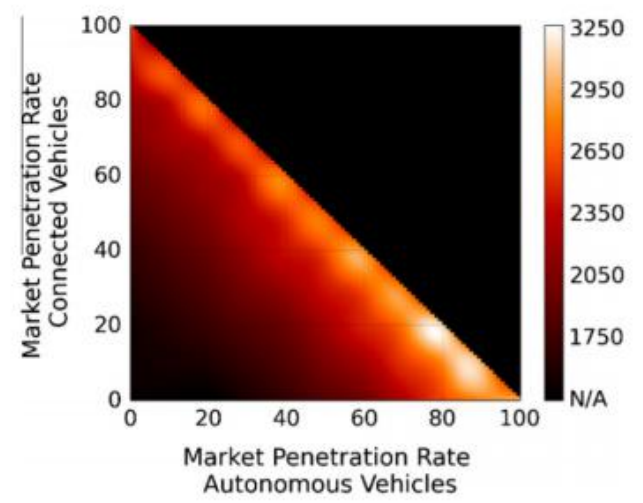

Figure 2 . Maximum throughput (veh/h/lane) at different market penetration rates of connected and autonomous vehicles for a platoon of regular, connected, and autonomous vehicles with infinite length [10].

The increase in road capacity needs to be quantitatively investigated. The increase in capacity is expected to be negligible $(1 \%-4 \%)$ if the CAV do not cooperate [12] and to be higher that $400 \%$ if efficient cooperation is achieved [13]. Lioris et al in [14] proved that connected vehicles can double or even triple the intersection capacity by simulation by using a memoryless queuing model with an on/off service process to simulate the behavior of $\mathrm{CAV}$ at an urban intersection and a case study in Los Angeles. However, it should be noticed that they choose the increase rate of demand and service ( $\mathrm{T}$ ) based on the empirical experience in a scenario where all vehicles are connected. As it is unrealistic to assume a fully connected condition at present, further research should be done to evaluate the value of $\mathrm{T}$ under different penetration rate of CAV. It is noticeable that most of the models proposed at present are not applicable for network of intersections. However, the road system in an urban area is often complex and contains various of intersections connected together. Therefore, it would be valuable to put effort in the model simulating more stochastic scenarios.

\section{Impacts on Environment}

The impact on environment is not the core consideration in the CAV development, but the influence could be significantly[15]. By introducing changes in travel demand, vehicle design and car following profiles, CAV will affect road energy consumption and greenhouse gas(GHG) emissions positively or negatively [16]. It is worth to notice that vehicles which are only automated but not connected may not be able to reduce energy consumption. A simulation found that an increase of energy consumption of an autonomous vehicle is $3 \%$ more than the traditional vehicle due to its following algorithms[17].In comparison, connected vehicles are better at predicting the behavior of preceding car by precise sensing ability and they can adjust their headway at a smooth motion. Wadud et al in [16] apply the ASIF framework proposed by Schipper in 2002 [18] to organize the potential mechanisms through which CAV affect the environment. They analysed the potential impacts in various illustrative scenarios and realized that the automation of vehicles might reduce the GHG emissions by nearly $50 \%$ or double it depending on which condition dominates.

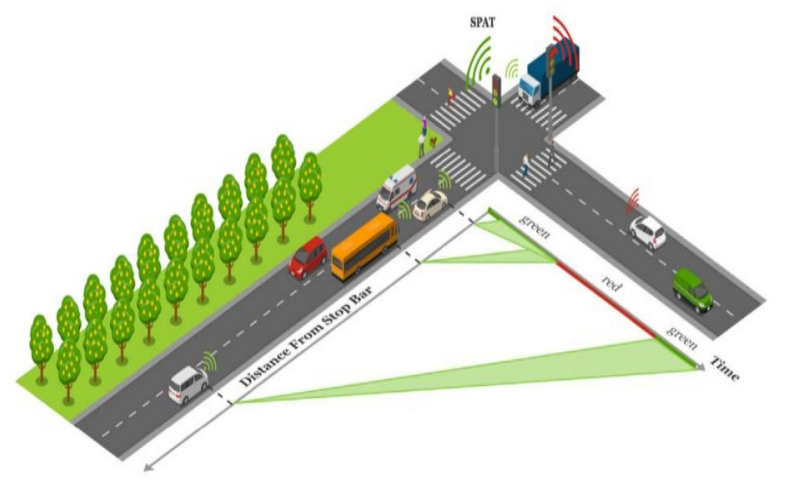

Figure 3. Schematic of eco-driving with SPAT preview [19]

As shown in the figure 3 above, The technology of Vehicle-to-Infrastructure(V2I) connectivity enables the Signal Phase and Timing (SPaT) to be broadcast to approaching vehicles, thus the vehicles are able to adjust their speed to make themselves arrive at the intersection at green light [19]. The models generated several researchers have supported the positive results (e.g. [20], [21]). However, it is noticeable that in the mixed traffic flow, the timely brake of CAV may be disruptive for the traffic environment. The micro models proposed is not 
sufficient to analyse this scenario and a smoother velocity profile should be developed considering the comfort of passengers and human drivers. Rakha and Kamalanathsarma in [22] investigated into this problem and by using a multi-stage dynamic model, they provided the speed trajectory which can save $19 \%$ energy.

How cars follow each other affects the fuel consumption significantly. Few branch of literature focus on the possible reduction in fuel use due to change in car following profiles. Li and Peng in [23] showed that around $30 \%$ energy can be saved by adopting a fueloptimal following strategy. A driver-vehicleinfrastructure cooperative framework which can save more than $20 \%$ energy is proposed by Qi in 2016 for electric vehicles [24]. Though framework is not designed for $\mathrm{CAV}$, the principle is valuable. The complex influence of communication delay is discussed by Ge and Orosz[25].

Though there have been papers analysing effect of CAV quantitatively, few of them focus on the quantitative impact on environment. It would be valuable to conduct more simulations to quantify the effect on environment based on new car-following profile with different penetration rate of CAV in the future. Moreover, even though vehicles are connected, how an automated vehicle and a human driver process the data of traffic environment can be significantly different. More research should be done to investigate how this difference would affect the car following and therefore the GHG emission.

\section{Impacts on Public Attitude}

Driving is a social activity and with the development of CAV technology, some social problems raised as well. Though it has been predicted that CAV will enable new user groups including children, disabled, elderly to enjoy the mobility, it does not mean that the society would be enthusiastic to CAV. Therefore, it is essential to monitor the public concerns on this technological development [26]. Several researches (e.g. [27], [28], [29]) have been conducted, trying to answer related questions: What will be the attitude of public towards CAV? Will people be willing to buy CAV? Will drivers be comfortable to share the road with CAV? The research in this area is multidisciplinary and usually involve psychological or social principles. These researches in a way suggest to which degree can public accept new advanced technology and the results therefore can be used in sociological fields. Tennant et al. conduct a cross national survey of drivers in 11 European countries with sample size of 1000 in each country in [30]. In this survey, more respondents give less enthusiastic attitude towards using CAV or driving alongside CAV and drivers who describe driving as an enjoyable activity tended to be more negative about AV. These results are consistent with the research conducted by European Commission in 2017 [31]. In terms of the willingness to use the CAV, the pricing of CAV plays an important role. $37 \%$ of the respondents in US indicated that they ' definitely would' or ' probably would' purchase a CAV as their next vehicle before learning the estimated price of $\$ 3000$ while this percentage dropped to $20 \%$ after they are told the prices [32]. The adoption of shared autonomous vehicles changed obviously under different pricing scenarios according to a survey conducted in US [33].

The problem of willingness of sharing road with CAV has not been given enough attention. It seems that there is lots of consideration on how autonomous vehicles would interact with human driven cars (as shown in the first section in this paper), while how human will response to CAV has not been studied thoroughly. It is always assumed as default that the interaction of human with autonomous vehicle is similar to interaction with traditional cars [30].

No matter how advanced the CAV technology has been, the benefits associated with CAV can not be achieved unless the public use, pay and buy CAV. One of the major concerns related to CAV is how to promote them and how well do people understand the autonomous vehicles. Moreover, CAV are not all fully automated and there are 6 levels of autonomous vehicles (Level 0 to Level 5) according to SAE [34]. It is predictable that there will be a long transition period when autonomous vehicles of heterogeneous levels share the road [35]. Therefore, several concerns arise: Do drivers understand their driving tasks with different levels of CAV? Do consumers hold a clear understanding of current taxonomy of CAV? These concerns are quite significant to the promotion and safety of CAV. The confusion among consumers might lead to safety issues like car accidents. A branch of papers have indicated that some of the names of level 2 autonomous vehicles which are available now make drivers over confident in cars [36,37]. For instance, the name 'Autopilot' used by Tesla makes considerable number of consumers to believe that they can take a nap when driving, which is very dangerous [36]. Speelt et. al. suggested that prevailing classification system of CAV is misleading to some degree and they thus created a new classification method which contained only two levels [38]. However, a two-level classification seems unrealistic as the autonomous driving system developed by car companies contain various levels of automation. Therefore, how to break consumers' confusion is an interesting topic in the future CAV promotion.

\section{Conclusion}

It is predictable that autonomous vehicles would be on our highways and streets in few years, making difference to the performance of our transportation network. The issues associated with the operation of the new transportation system will be challenging and comprehensive. From pure mathematical framework, to models incorporating real world conditions, to social surveys, multiple methods of research have been used to anticipate the impacts of CAV. By reviewing literature related to the influence on road capacity, environment and public attitude, it is found that the complexity of these issues is huge. Though a general positive future is anticipated by most academics, it is hard to give a conclusion on either how much the road capacity would 
change or how the GHG emission would change with penetration of CAV. More efforts should be put in modelling network of intersections and changes of human drivers after introducing CAV to analyse the potential impacts quantitatively. It is hard to say that the public hold a very positive attitude towards CAV based on current surveys. To promote the CAV among the public, a simpler classification system of AV might be helpful. Policy makers should also pay attention to the public attitude and role of responsibility for human drivers with various levels of CAV to adjust the business or road control policies.

\section{References}

1. McCarthy, N.(2015). Connected cars by the numbers. Forbes.

2. Litman, T. (2017). Autonomous vehicle implementation predictions. Victoria Transport Policy Institute.

3. Litman, T., 2015. Autonomous vehicle implementation predictions implications for transport planning. Transportation Research Board 94th Annual Meeting.

4. Chen, D., Ahn, S., Chitturi, M., \& Noyce, D. (2017). Towards vehicle automation: Roadway capacity formulation for traffic mixed with regular and automated vehicles. Transportation Research. Part B, Methodological, 100, 196.

5. Gong, Shen \& Du, 2016. Constrained optimization and distributed computation based car following control of a connected and autonomous vehicle platoon. Transportation Research Part B, 94, pp.314-334.

6. Talebpour, \& Mahmassani. (2016). Influence of connected and autonomous vehicles on traffic flow stability and throughput. Transportation Research Part C,71, 143-163.

7. Young, S.H. \& The George Washington University. Engineering Mgt Systems Engineering., degree granting institution, 2016. A Model-Based Framework for Predicting Autonomous Unmanned Ground Vehicle System Performance.

8. Ghiasi et al., 2017. A mixed traffic capacity analysis and lane management model for connected automated vehicles: A Markov chain method. Transportation Research Part B, 106, pp.266 - 292.

9. Levin, \& Boyles. (2016). A multiclass cell transmission model for shared human and autonomous vehicle roads. Transportation Research Part C, 62, 103-116.

10. Yoon, J.-woo \& Kim, B.-woo, 2016. Vehicle position estimation using nonlinear tire model for autonomous vehicle. Journal of Mechanical Science and Technology, 30(8), pp.3461-3468.

11. Shladover, S., Su, D., \& Lu, X. (2016). Impacts of Cooperative Adaptive Cruise Control on Freeway Traffic Flow. Transportation Research Record, 2324(2324), 63-70.

12. Fernandes, P., \& Nunes, U. (2012). Platooning With IVC-Enabled Autonomous Vehicles: Strategies to
Mitigate Communication Delays, Improve Safety and Traffic Flow. IEEE Transactions on Intelligent Transportation Systems, 13(1), 91-106.

13. Lioris, Pedarsani, Tascikaraoglu, \& Varaiya. (2017). Platoons of connected vehicles can double throughput in urban roads. Transportation Research Part C, 77(C), 292-305.

14. Vahidi, \& Sciarretta. (2018). Energy saving potentials of connected and automated vehicles. Transportation Research Part C, 95, 822-843.

15. Wadud, Z., MacKenzie, D., \& Leiby, P. (2016). Help or hindrance? The travel, energy and carbon impacts of highly automated vehicles. Transportation Research, Part A, 86, 1-18.

16. Mersky, \& Samaras. (2018). Corrigendum to "Fuel economy testing of autonomous vehicles" [Transp. Res. Part C: Emerging Technol. 65 (2016) 31-48]. Transportation Research Part C, 87, 212-217.

17. Schipper, L. (2002). Sustainable urban transport in the 21st century. A new agenda. Transportation Research Record, (1792), 12-19.

18. Miller, S.A. \& Heard, B.R., 2016. The Environmental Impact of Autonomous Vehicles Depends on Adoption Patterns. Environmental science \& technology, 50(12), pp.6119-6121.

19. Mahler, G., \& Vahidi, A. (2014). An Optimal Velocity-Planning Scheme for Vehicle Energy Efficiency Through Probabilistic Prediction of Traffic-Signal Timing. IEEE Transactions on Intelligent Transportation Systems, 15(6), 25162523.

20. Rakha, Ahn, Moran, Saerens, \& Bulck. (2011). Virginia Tech Comprehensive Power-Based Fuel Consumption Model: Model development and testing. Transportation Research Part D, 16(7), 492503.

21. Kamalanathsharma, R., \& Rakha, H. (2013). Multistage dynamic programming algorithm for ecospeed control at traffic signalized intersections. 16th International IEEE Conference on Intelligent Transportation Systems (ITSC 2013), 2094-2099.

22. Li, S., \& Huei Peng. (2011). Strategies to minimize fuel consumption of passenger cars during carfollowing scenarios. Proceedings of the 2011 American Control Conference, 2107-2112.

23. Qi, X. (2016). Next Generation Intelligent DriverVehicle-Infrastructure Cooperative System for Energy Efficient Driving in Connected Vehicle Environment. 214.

24. Ge, \& Orosz. (2014). Dynamics of connected vehicle systems with delayed acceleration feedback. Transportation Research Part C, 46(C), 46-64.

25. Becker, F., \& Axhausen, K. (2017). Literature review on surveys investigating the acceptance of automated vehicles. Transportation, 44(6), 12931306.

26. Lamotte, R., de Palma, A. \& Geroliminis, N., 2017. On the use of reservation-based autonomous vehicles for demand management. Transportation Research. Part B, Methodological, 99, p.205.

27. Grush, B. \& Niles, J., 2018. The end of driving : transportation systems and public policy planning 
for autonomous vehicles, Amsterdam, Netherlands: Elsevier.

28. Hudson, Orviska \& Hunady, 2019. People' s attitudes to autonomous vehicles. Transportation Research Part A, 121, pp.164 - 176.

29. Schipper, L. (2002). Sustainable urban transport in the 21st century. A new agenda. Transportation Research Record, (1792), 12-19.

30. European Commission. (2017). Special Eurobarometer 460: Attitudes towards the impact of digitisation and automation on daily life.

31. J.D.Power.(2012). Vehicle Owners Show Willingness to Spend on Automotive Infotainment Features, Westlake Village.

32. Banal, Kockelman, \& Singh. (2016). Assessing public opinions of and interest in new vehicle technologies: An Austin perspective. Transportation Research Part C, 67(C), 1-14.

33. SAE International. 2019. Taxonomy and Definitions for Terms Related to Driving Automation Systems for On-Road Motor Vehicles. [ONLINE] Available at: https://www.sae.org/standards/content/j3016_20180 6/. [Accessed 20 July 2019].

34. Prakah-Asante, K.O., Strumolo, G.S. \& Curry, R., 2018.

35. IIHS. 2019. New studies highlight driver confusion about automated systems. [ONLINE] Available at: https://www.iihs.org/news/detail/new-studieshighlight-driver-confusion-about-automatedsystems. [Accessed 20 July 2019].

36. IIHS. 2019. What $\mathrm{s}$ in a name? Drivers, perceptions of the use of five SAE Level 2 driving automation systems. [ONLINE] Available at: https://www.iihs.org/topics/bibliography/ref/2190. [Accessed 20 July 2019].

37. 10th International Driving Symposium on Human Factors in Driver Assessment, Training, and Vehicle Design. 2019. Consumer confusion with levels of vehicle automation.[ONLINE]Available at: https://rivingassessment.uiowa.edu/sites/driving assessment.uiowa.edu/files/wysiwyg_uploads/combi nedprogramschedulefiles_6_10_19_0.pdf.

[Accessed 20 July 2019]. 